\title{
Evaluation of changes in sexual response and factors influencing sexuality during pregnancy among Nigerian women in Jos, Nigeria
}

\author{
Stephen A. Anzaku ${ }^{1}$, Emmanuel A. Ogbe ${ }^{2}$, George I. Ogbu ${ }^{3}$, Bassey E. Edem ${ }^{4}$, \\ Stephen D. Ngwan
}

\author{
${ }^{1}$ Department of Obstetrics and Gynaecology, College of Health Sciences, Bingham University, Jos campus, Jos, \\ Nigeria \\ ${ }^{2}$ Department of Obstetrics and Gynaecology, Dalhatu Araf Specialist Hospital, Lafia, Nigeria \\ ${ }^{3}$ Department of Obstetrics and Gynaecology, Federal Medical Centre Keffi, Nigeria \\ ${ }^{4}$ Department of Anaesthesia, Benue State University Teaching Hospital, Makurdi, Nigeria \\ ${ }^{5}$ Department of Obstetrics and Gynaecology, Federal Medical Centre Makurdi, Nigeria
}

Received: 22 August 2016

Accepted: 17 September 2016

\author{
*Correspondence: \\ Dr. Stephen A. Anzaku, \\ E-mail: steveanzaku@gmail.com
}

Copyright: () the author(s), publisher and licensee Medip Academy. This is an open-access article distributed under the terms of the Creative Commons Attribution Non-Commercial License, which permits unrestricted non-commercial use, distribution, and reproduction in any medium, provided the original work is properly cited.

\begin{abstract}
Background: Pregnancy is characterized by physical, hormonal and psychological changes that could influence women's sexuality. The study aimed at ascertaining changes in the women's sexual domains as well as factors affecting their sexual responses.

Methods: A total of 177 healthy heterosexual pregnant Nigerian women at term and in stable marital relationships were included in the study. Authors' designed structured questionnaire featuring socio-demographic and obstetric characteristics as well as assessment of their sexual desire, arousal, orgasm, sexual satisfaction and pain compared to the pre-pregnancy period was used to collect the information. Data was analyzed using SPSS version 16 for windows. Results: Mean age of the women was $30.9 \pm 4.7$ years. Majority of them reported decline in sexual desire, arousal, frequency of orgasm and sexual satisfaction compared to the pre-pregnancy period. Reduce sexual desire was marked in the first trimester but sexual desire peaked in second trimester. Women aged $\geq 31$ years were four times more likely to experience increase frequency of orgasm (OR 4.0, 95\% CI $1.9-8.7, \mathrm{P}=0.02)$ while those with tertiary education (OR 2.2, 95\% CI 1.1 - 4.2, P = 0.02) and unplanned pregnancy (OR 2.4, 95\% CI 1.8 - 5.0, P = 0.04) were more likely to experience decreased sexual satisfaction compared to the pre-pregnancy period.

Conclusions: Pregnancy is associated with decline in all domains of female sexual response cycle among the women. Older maternal age positively impacts on frequency of attainment of orgasm while tertiary educational level and unplanned pregnancy negatively affect their sexual satisfaction during pregnancy.
\end{abstract}

Keywords: Pregnancy, Sexual function, Changes in sexuality, Nigeria

\section{INTRODUCTION}

Sexual relationship of an expectant mother is of immense emotional and psychological significance as it may be a source factor that can enhance her quality of life or that of the couple. Pregnancy has been defined as a difficult period of life for women punctuated by physical and emotional changes that affect their sexual lives.,
Deterioration in pregnant women's sexuality do not only affect their physical and psychological health but the health of the family as a whole. Hormonal changes in pregnancy and non-hormonal influences such as emotional, socio-economic, cultural and religious beliefs are thought to be associated with these changes in sexual behavior among pregnant women., 
Pregnant women's sexuality may also be affected as a result of physical complaints they experience including fatigue, backache, frequent urination, heartburn, breast tenderness and headaches. ${ }^{4,5}$ Frequently, there is decrease sexual desire and arousal due to nausea and vomiting in the first trimester, fatigue and physical discomfort in the third trimester as well as psychological factors. ${ }^{1,4,6,7}$ The fact that coital frequency and sexual satisfaction are influenced by sexual desire and arousal makes it reasonable to infer that these two sexual domains may also be affected adversely among pregnant women.,

Duration of intercourse and ability to achieve orgasm has been noted to decrease during second and third trimesters of pregnancy compared to the pre-pregnancy period. ${ }^{10}$ Pregnant women usually have unpleasant experiences during sexual intercourse including dyspareunia probably due to changes in vaginal lubrication and difficulties in adopting comfortable coital positions. ${ }^{4,5,10-12}$

Hence, it seems sexual relationship of expectant mothers is one of the vulnerable areas during pregnancy and they may unavoidably develop changes in their sexual responses as a result of various discomforts and complaints they experience. Women often do not complain about sexual changes during pregnancy as open discussion of sexual issues is often regarded as a taboo topic in most cultures including ours. This study sought to ascertain changes in our women sexuality during pregnancy compared to the pre-pregnancy period as well as factors influencing the various sexual domains in the sexual response cycle among this group of Nigerian women.

\section{METHODS}

This was a descriptive cross-sectional study carried out among healthy heterosexual pregnant Nigerian women at term ( $\geq 37$ weeks) between January and June 2015 in the antenatal clinic of Bingham University Teaching Hospital, Jos. The women were approached individually without a third party and in the absence of their spouses and were recruited by convenient sampling method. Pregnant women with feto-maternal complication(s) or ay medical morbidity that may affect their sexual relationship were excluded from the study. All eligible women attending antenatal care during the study period and consented to participate in the research were included in the study.

Pre-tested questionnaire designed by the researchers were administered on the women and they were filled anonymously and returned. For the few that could not self-administer the questionnaires, a trained female Nurse administered and filled the questionnaires appropriately. Inquiries were made about their socio-demographic and obstetric features as well as rating of their sexual desire compared to the pre-pregnancy period. Rating was done with selection of options as "same", "decrease", and "increase". Also rated compared to the pre-pregnancy period were other domains in the female sexual response cycle including sexual arousal, attainment of sexual climax (orgasm), and sexual satisfaction. Pain during sexual intercourse (dyspareunia) and average two weekly coital frequencies were also ascertained. Dyspareunia was assessed as "none", "occasional" and "always".

The information collected from the patients was entered into SPSS version 16 for windows (SPSS Inc., Chicago, IL, USA). Data was analysed as simple percentages and mean \pm standard deviation (SD). Chi square test or Fisher exact test (when expected value was <5) where appropriate was used to ascertain socio-demographic and obstetric factors affecting various domains of the female sexual response cycle. A P value of $<0.05$ was considered statistically significant. Ethical approval for the study was obtained from the Human Research and Ethics Committee of Bingham University Teaching Hospital, Jos, Nigeria.

\section{RESULTS}

Table 1: Socio-demographic and obstetric features of the women.

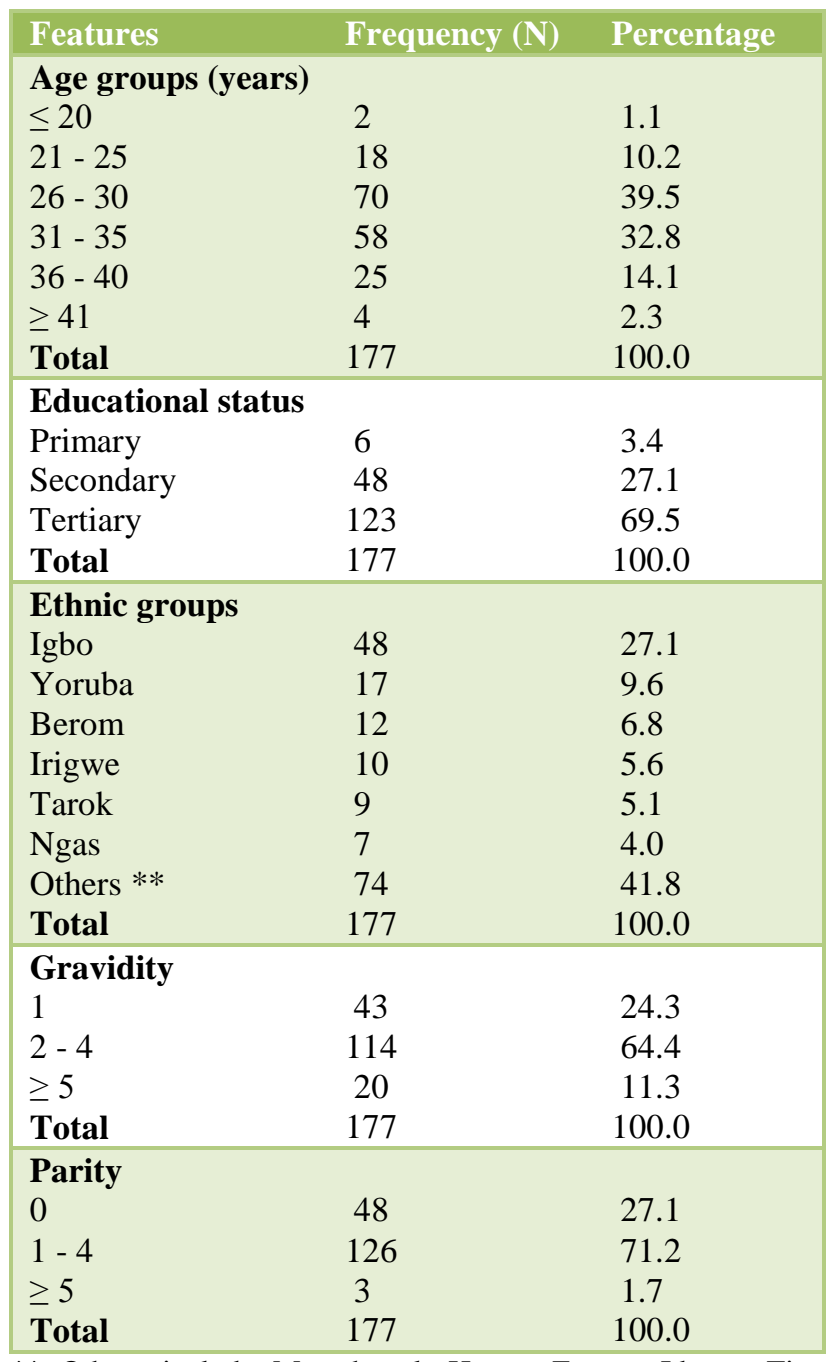

** Others include Mwaghavul, Hausa, Eggon, Idoma, Tiv, Mupun, Afizere, Tangale, Ron, Mupun, Rukuba, Igala, Mushere 
Table 2: Changes in sexuality during pregnancy compared to pre-pregnancy among the study population.

\begin{tabular}{|llll|}
\hline $\begin{array}{l}\text { Sexual } \\
\text { domain }\end{array}$ & $\begin{array}{l}\text { Increase N } \\
(\%)\end{array}$ & $\begin{array}{l}\text { Decrease } \mathbf{N} \\
(\%)\end{array}$ & $\begin{array}{l}\text { No } \\
\text { change N } \\
(\%)\end{array}$ \\
\hline $\begin{array}{l}\text { Sexual } \\
\text { desire }\end{array}$ & $28(15.8)$ & $100(56.5)$ & $49(27.7)$ \\
\hline $\begin{array}{l}\text { Sexual } \\
\text { arousal }\end{array}$ & $34(19.2)$ & $92(52.0)$ & $51(28.8)$ \\
\hline $\begin{array}{l}\text { Frequency } \\
\text { of orgasm }\end{array}$ & $36(20.3)$ & $84(47.5)$ & $57(32.2)$ \\
\hline $\begin{array}{l}\text { Sexual } \\
\text { satisfaction }\end{array}$ & $27(15.2)$ & $89(50.3)$ & $61(34.5)$ \\
\hline
\end{tabular}

A total of 177 (91.7\%) pregnant women out of 193 from 35 different ethnic groups in Nigeria correctly completed the questionnaires. The mean age of the women was 30.9 \pm 4.7 years with a range of $20-42$ years. The mean gravidity and parity were $2.69 \pm 1.48$ and $1.44 \pm 1.26$ respectively with range of parity between $0-7$. Most of the women $[170(96.0 \%)]$ were Christians while 7 (4.0\%) were Muslims. Majority of the women had planned pregnancies [128(72.3\%] while $49(27.7 \%)$ admitted that their pregnancies were unplanned. Twenty seven $(15.3 \%)$ of them were HIV positive while 150 (84.7\%) were nonreactive to HIV infection. Table 1 shows the sociodemographic and obstetric features of the study population.

Table 3: Factors influencing changes in sexual desire and arousal among the women.

\begin{tabular}{|llll|}
\hline Sexual domain/Factors & Odds ratio & $95 \%$ Confidence interval & P-value \\
\hline Decrease sexual desire & & & \\
Maternal age $\geq 31$ (years) & 0.53 & $0.23-1.24$ & 0.16 \\
Tertiary educational status & 1.18 & $0.62-2.24$ & 0.62 \\
Primigravidity & 1.64 & $0.79-3.28$ & 0.19 \\
Nulliparity & 1.41 & $0.71-2.77$ & 0.33 \\
unplanned pregnancy & 0.60 & $0.30-1.19$ & 0.15 \\
Positive HIV status & 1.05 & $0.46-2.39$ & 0.92 \\
Increase sexual desire & & & \\
Maternal age $\geq 31$ (years) & 1.21 & $0.39-3.79$ & $1.00 \neq$ \\
Tertiary educational status & 0.91 & $0.38-2.17$ & 0.84 \\
Primigravidity & 1.60 & $0.66-3.87$ & 0.29 \\
NulliParity & 1.96 & $0.84-4.56$ & 0.12 \\
unplanned pregnancy & 1.93 & $0.69-5.40$ & 0.21 \\
Positive HIV status & 0.60 & $0.22-1.67$ & $0.39 \neq$ \\
\hline Decrease sexual arousal & & & \\
Maternal age $\geq 31$ (years) & 0.73 & $0.32-1.62$ & 0.44 \\
Tertiary educational status & 1.39 & $0.73-2.64$ & 0.32 \\
Primigravidity & 1.23 & $0.62-2.45$ & 0.56 \\
Nulliparity & 1.13 & $0.58-2.19$ & 0.72 \\
unplanned pregnancy & 0.67 & $0.34-1.30$ & 0.24 \\
Positive HIV status & 0.84 & $0.37-1.92$ & 0.69 \\
Increase sexual arousal & & & \\
Maternal age $\geq 31$ (years) & 0.83 & $0.33-2.13$ & 0.70 \\
Tertiary educational status & 0.60 & $0.28-1.26$ & 0.18 \\
Primigravidity & 1.15 & $0.51-2.61$ & 0.74 \\
Nulliparity & 1.32 & $0.60-2.89$ & 0.49 \\
Unplanned pregnancy & 1.30 & $0.57-3.00$ & 0.54 \\
Positive HIV status & 0.74 & $0.29-1.92$ & 0.52 \\
\hline -Fiter & & &
\end{tabular}

$\neq$ - Fisher exact test

On assessing the women two weekly sexual frequency, about $35.6 \%(63 / 177)$ of them reported that sometimes they don't engage in any sexual intercourse, $45(25.4 \%)$ reported average of one episode, $38(21.4 \%)$ reported 2 episodes, $20(11.3 \%)$ reported average of 3 episodes while $11(6.2 \%)$ reported 4 or more episodes. The women were also asked to rate their overall frequency of sexual intercourse during the pregnancy compared to the prepregnancy period. Ninety eight $(55.4 \%)$ of them reported decrease sexual frequency, $51(28.8 \%)$ reported no change while $28(15.8 \%)$ reported increase frequency of sexual intercourse during pregnancy. Inquiry about painful sexual intercourse (superficial and deep dyspareunia) experienced during the pregnancy was also 
done. Seventy two (40.7\%) reported no episode, 97 $(54.8 \%)$ reported occasional episodes while $8(4.5 \%)$ of them reported that they always had dyspareunia.

Rating of the different domains of the female sexual response cycle was ascertained among the women. The results revealed overall decrease in the various sexual domains during pregnancy compared to the prepregnancy period (Table 2). Rating of the women's sexual desire as "lowest", "medium", and "highest" according to trimesters of pregnancy showed that most of them experienced highest state of sexual desire in the second trimester. Majority [114 (64.4\%)] reported that sexual desire was lowest during the first trimester, 33 $(18.6 \%)$ rated as medium while $30(17.0 \%)$ rated it as highest. During the second trimester, $37(20.9 \%)$ rated their sexual desire as lowest, $59(33.3 \%)$ indicated that it was medium while $81(45.8 \%)$ reported highest state of sexual desire. In the third trimester, $56(31.6 \%)$ revealed lowest level of sexual desire, $72(40.7 \%)$ indicated as medium while $49(27.7 \%)$ reported highest state of sexual desire compared to other trimesters.

Table 4: Factors influencing changes in orgasm and sexual satisfaction among the women.

\begin{tabular}{|c|c|c|c|}
\hline Sexual domain & Odds ratio & 95\% Confidence interval & P-value \\
\hline \multicolumn{4}{|c|}{ Decrease frequency of orgasm } \\
\hline Maternal age $\geq 31$ (years) & 1.35 & $0.75-2.44$ & 0.32 \\
\hline Tertiary educational status & 1.19 & $0.63-2.26$ & 0.60 \\
\hline Primigravidity & 1.08 & $0.54-2.14$ & 0.84 \\
\hline Nulliparity & 1.03 & $0.53-1.99$ & 0.94 \\
\hline unplanned pregnancy & 0.82 & $0.43-1.59$ & 0.56 \\
\hline Positive HIV status & 0.97 & $0.43-2.10$ & 0.94 \\
\hline \multicolumn{4}{|c|}{ Increase frequency of orgasm } \\
\hline Maternal age $\geq 31$ (years) & 4.01 & $1.90-8.70$ & 0.02 \\
\hline Tertiary educational status & 0.85 & $0.39-1.85$ & 0.68 \\
\hline Primigravidity & 1.50 & $0.67-3.37$ & 0.33 \\
\hline Nulliparity & 1.46 & $0.66-3.21$ & 0.35 \\
\hline unplanned pregnancy & 0.99 & $0.44-2.25$ & 0.96 \\
\hline Positive HIV status & 0.69 & $0.26-1.77$ & 0.44 \\
\hline \multicolumn{4}{|l|}{ Decrease sexual satisfaction } \\
\hline Maternal age $\geq 31$ (years) & 0.98 & $0.54-1.76$ & 0.94 \\
\hline Tertiary educational status & 2.17 & $1.13-4.19$ & 0.02 \\
\hline Primigravidity & 0.93 & $0.47-1.84$ & 0.83 \\
\hline Nulliparity & 0.78 & $0.40-1.52$ & 0.47 \\
\hline unplanned pregnancy & 2.41 & $1.82-5.04$ & 0.04 \\
\hline Positive HIV status & 0.93 & $0.41-2.11$ & 0.86 \\
\hline \multicolumn{4}{|l|}{ Increase sexual satisfaction } \\
\hline Maternal age $\geq 31$ (years) & 0.74 & $0.32-1.68$ & 0.47 \\
\hline Tertiary educational status & 0.41 & $0.18-1.07$ & 0.34 \\
\hline Primigravidity & 1.12 & $0.43-2.83$ & 0.73 \\
\hline Nulliparity & 1.16 & $0.47-2.85$ & 0.75 \\
\hline unplanned pregnancy & 1.11 & $0.44-2.82$ & 0.83 \\
\hline Positive HIV status & 0.57 & $0.21-1.58$ & 0.28 \\
\hline
\end{tabular}

Assessment of the impacts of socio-demographic and obstetric factors on the various domains of the female sexual response cycle (sexual desire, sexual arousal, orgasm) as well as sexual satisfaction was done. Women aged $\geq 31$ years were four times more likely to experience increased episodes of orgasm compared to the prepregnancy period (OR 4.0, 95\% CI $1.9-8.7, \mathrm{P}=0.02)$ while those with tertiary education (OR $2.2,95 \%$ CI $1.1-$ $4.2, \mathrm{P}=0.02)$ and unplanned pregnancy (OR 2.4, 95\% CI $1.8-5.0, \mathrm{P}=0.04)$ were more likely to experience decrease sexual satisfaction during pregnancy. Table 3 and 4 show the outcomes of the influences of socio- demographic and obstetric factors on the domains of the women's sexual response cycle.

\section{DISCUSSION}

Female sexual life, changes and responses during pregnancy are rarely discussed between couples and their midwives and doctors. It is widely acknowledged that pregnancy influences sexual behavior of women including frequency of sexual intercourse and the domains of the female sexual response cycle. ${ }^{7,10,13}$ Hormonal, physical and psychological changes in 
pregnancy impact on women's sexuality as they provoke and promote changes in sexual response and activities. ${ }^{14}$

This study revealed overall decrease in all domains of the female sexual response cycle in majority of the study population and this has been corroborated by other researchers. ${ }^{5,15-17}$ Sexual relationships during pregnancy can enhance couples' bonding and quality of life and the need to know these potential changes in sexuality during pregnancy by them is crucial so as to avoid marital disharmony. Hence, the importance of health workers involved in the care of pregnant women in any clinical setting in providing counselling regarding sexual aspects of pregnancy including changes that may occur cannot be over-emphasized.

More than half of the women reported decline in sexual desire during pregnancy compared to the pre-pregnancy period while the rest reported either increase or no change in their interest for sexual intercourse. In addition, about two-thirds of the women reported that decline in sexual desire was highest or most marked during the first trimester but peaked in the second trimester. These findings have also been corroborated by other researchers from different antenatal populations. ${ }^{14,17-19}$ The decline in sexual desire especially in the first trimester has been attributed to hormonal alterations leading to morning sickness and its attendant discomforts, breast tenderness as well as diminished clitoral sensation and orgasmic disorders. ${ }^{15,20,21}$ The tendency towards improvement in sexual desire in the second trimester as noted in this study may be due to reduction of the symptoms and physical discomforts in the first trimester. The fact that some women experienced no change or increase sexual desire during pregnancy in this study has also been reported elsewhere. $^{22,23}$ Variations in sexual desire among pregnant women may be a pointer to complex interactions of factors affecting this sexual domain including social and cultural factors. ${ }^{18}$ This may explain the finding of our study as the study population was from 35 different ethnic groups spread across Nigeria.

Our research also revealed variable states of sexual arousal among the women compared to the prepregnancy period with more than half of them reporting decrease arousal, about $20 \%$ disclosed increase while the rest reported no change in sexual arousal. Decrease in sexual arousal and lubrication have been reported among majority of pregnant Taiwanese and Iranian women ${ }^{13,17}$ but contrary to findings elsewhere. ${ }^{4,10}$ In general, there is vaso-congestion of vaginal tissues during pregnancy as a result of increase blood circulation and this leads to increase sexual arousal and lubrication. ${ }^{3,18}$ However, the variable states of sexual arousal among pregnant women may also be attributed to different factors in and around the pregnant woman that may influence her sexuality.

With respect to orgasm among the women, most women reported decrease rate of attainment of orgasm while about one-third disclosed increase frequency of orgasm compared to the pre-pregnancy period. Decrease in rate of orgasm among pregnant women has been reported by several researchers but it was noted that this improves as gestational age increases. ${ }^{10,13,17,24}$ This may be attributed to increasing pelvic vaso-congestion in the course of pregnancy thereby enhancing vaginal sensation and facilitating attainment of orgasm.

Pregnancy has also been noted as a period of decrease sexual satisfaction among women ${ }^{16,25}$ and this was noted in about $50 \%$ of our study population. This may be attributed to decrease in frequency and length of sexual intercourse which occur during pregnancy ${ }^{17,22,23,26}$ with resultant poor achievement of orgasm and sexual satisfaction. Also, the state of emotional relationship with the husband has also been noted to influence sexual satisfaction during pregnancy. ${ }^{27}$ Reported experience of dyspareunia among this study population may also have contributed to the decrease sexual satisfaction among the women.

Several researchers have also reported negative impact of pregnancy on incidence of painful sexual intercourse. ${ }^{16,17,24}$ This was also noted in this study where most of the women either reported occasional episodes of dyspareunia or dyspareunia at every sexual encounter compared to the pre-pregnancy period. This increase incidence of dyspareunia during pregnancy has been attributed to anatomical changes that occur in pregnancy as well as hypertrophy of pelvic viscera. ${ }^{28}$

Several socio-demographic and obstetric factors have been reported to have positive or negative influences on sexual function and response of pregnant women. In this study, women aged 31 years and above were most likely to experience increase frequency of attainment of orgasm during pregnancy compared to the pre-pregnancy period but maternal age had no impact on their sexual desire, arousal and sexual satisfaction. The finding of older age group having positive influence on rate of attainment of orgasm is contrary to that reported by other researchers who noted that older aged women reported negative influences on their sexual function during pregnancy. ${ }^{1,29,30}$ However, a study in Turkey demonstrated that age does not influence sexual behavior and responses of pregnant women ${ }^{4}$ as also noted in other sexual domains of the female sexual response cycle in this study. These different findings of the impact of maternal age on female sexuality may be attributed to variations in cultural practices of the women and so its influence on their sexual behavior and response.

Educational level of women is also said to influence their sexual function and response during pregnancy. ${ }^{30,31}$ This study shows that higher level of education negatively impacts on sexual satisfaction among the women but not on other sexual domains. This is however contrary to a report from Egypt that showed women with lower level of education were more likely to experience disturbed sexual function during pregnancy. ${ }^{32}$ These varied reports may be a reflection of the impacts of diverse factors on female sexuality among different obstetric populations. 
Our study also found that gravidity and parity have no impact on the domains of the female sexual response cycle among the women. This finding is corroborated by a study conducted in an Egyptian obstetric population ${ }^{32}$ but contrary to findings elsewhere indicating that primiparity negatively impacts on the sexual function of pregnant women. ${ }^{33,34}$

These inconsistent findings by researchers may be attributed to differences in study designs and degree of impacts of social, cultural, psychological and relationship factors on sexuality of pregnant women in the different obstetric populations studied.

Also positive HIV status had no influence on the women's sexuality but unplanned pregnancy has negative influence on their sexual satisfaction. The finding of noninfluence of HIV status on the sexual domains of the women may be attributed to the fact that all the HIV positive were on highly active anti-retroviral drugs (HAART) and probably the husbands especially the discordant partners were using barrier method of contraceptive to protect themselves and this will make the couples to be less worried about the disease while engaging in sexual intercourse. Also, possible psychological impact of unplanned pregnancy on the women may be responsible for the decrease sexual satisfaction among them.

This study has some limitations worth stating. Recall bias among the women probably affected the outcomes of the study and the fact that it was conducted in a Teaching Hospital may not be a true representation of what is obtainable in the general society. However, the study for the first time highlighted changes in female sexuality as well as the factors affecting it in this obstetric population.

In conclusion, Pregnancy is associated with decline in the domains of the female sexual response cycle among these Nigerian women. Older maternal age positively impacts on frequency of attainment of orgasm while tertiary educational level and unplanned pregnancy negatively affect their sexual satisfaction. Counselling of couples to be informed about changes in female sexual responses in pregnancy is imperative so as to avoid marital disharmony during the period.

\section{ACKNOWLEDGEMENTS}

Authors would like to acknowledge Mrs. Mary David Atu for distributing the questionnaires to the women and interviewing those that were not literate.

Funding: No funding sources Conflict of interest: None declared

Ethical approval: The study was approved by the Institutional Ethics Committee

\section{REFERENCES}

1. Gokyildiz S, Kizilkaya N. The effects of pregnancy on sexual life. J Sex Marital Ther. 2005;31:201-5.

2. Sossah L. Sexual behavior during pregnancy: a descriptive correlational study among pregnant women. European Journal of Research in Medical Sciences. 2014;2(1):16-27.

3. Bitzer J, Adler J. Sexuality during pregnancy and the post-partum period. Journal of sex education and therapy. 2000;25(1):49-58.

4. Eryilmaz G, Ege E, Zincir H. Factors affecting sexual life during pregnancy in eastern Turkey. Gynecol Obstet Invest. 2004;57:103-8.

5. Pauls RN, Occhino JA, Dryfhout VL. Effects of pregnancy on female sexual function and body image: A prospective study. J Sex Med. 2008;5:1915-22.

6. Millheiser L. Female sexual function during pregnancy and postpartum. J Sex Med. 2012;9:6356.

7. Aslan G, Aslan D, Kizilyar A, Ispahi C, Esen A. A prospective analysis of sexual functions during pregnancy. Int J Impot Res. 2005;17:154-7.

8. Basson R. Human sex-response cycles. J Sex Marital Ther. 2001;27:33-43.

9. Pauleta JR, Pereire NM, Graca LM. Sexuality during pregnancy. J Sex Med. 2010;7:136-42.

10. Von Sydow KV. Sexuality during pregnancy and after childbirth: A meta-content analysis of 59 studies. J Psychosom Res. 1999;47:27-49.

11. Trutnovsky G, Haas J, Lang U, Petru E. Women's perception of sexuality during pregnancy and after childbirth. Aust N Z J Obstet Gynaecol. 2006;46:283-7.

12. Fok WY, Chan LY, Yuen PM. Sexual behaviour and activity in Chinese pregnant women. Acta Obstet Gynaecol Scand. 2005;84:934-8.

13. Lee JT. The meaning of sexual satisfaction in pregnant Taiwanese women. J Midwifery Womens Health. 2002;47:278-86.

14. Khamis MA, Mustafa MF, Mohamed SN. Influence of gestational period on sexual behavior. J Egypt Public Health Assoc. 2007;82(1\&2):65-90.

15. Erol B, Sanli O, Korkmaz D, Seyhan A, Akman T, Kadioglu A. A cross-sectional study of female sexual function and dysfunction during pregnancy. J Sex Med. 2007;4:1381-7.

16. Aboyeji PA, Ijaiya MA. Sexuality and sexual activities in pregnant Nigerian women in Ilorin. Sexual Health Matters. 2005;6(3):65-8.

17. Jamali S, Mosalanejad L. Sexual dysfunction in Iranian pregnant women. Iran J Reprod Med. 2013;11(6):479-86.

18. Jones C, Chan C, Farine D. Sex in pregnancy. Canadian Med Assoc J. 2011;183(7):815-8.

19. Corbacioglu EA, Akca A, Akbayir O, Goksedef BP, Bakir VL. Female sexual function and associated factors during pregnancy. J Obstet Gynaecol Res. 2013;39(6):1165-72. 
20. Pessina MA, Hoyt RF Jr, Goldstein I, Traish AM. Differential regulation of the expression of estrogen, progesterone, and androgen receptorsby sex steroid hormones in the vagina: immunohistochemical studies. J Sex Med. 2006;3(5):804-14.

21. Warnock JK, Clayton A, Croft H, Segraves R, Biggs FC. Comparison of androgens in women with hypoactive sexual desire disorders: those on combined oral contraceptives (COCs) Vs those not on COCs. J Sex Med. 2006;3(5):878-82.

22. Naim M, Bhotto E. Sexuality during pregnancy in Pakistani women. J Pak Med Assoc. 2000;50(1):3844.

23. Orji EO, Ogunlola IO, Fasuuba OB. Sexuality among pregnant women in South West Nigeria. J Obstet Gynaecol. 2002;22:166-8.

24. Johnson CE. Sexual health during pregnancy and the postpartum. J Sex Med. 2011;8(5):1267-84.

25. Kontayannis M, Katsetos C, Panagopoulos P. Sexual intercourse during pregnancy. Health Science Journal. 2012;6(1):82-7.

26. Anzaku AS, Okoye FN, Bulus AD, Edem BE. Frequency, perceptions and complications of sexual activity during pregnancy among a group of Nigerian women. IAIM. 2015;2(6):54-63.
27. Dejudicibus MA, McCabe MP. Psychological factors and the sexuality of pregnant and postpartum women. J Sex Res. 2002;39(2):94-103.

28. Reamy K, White SE, Daniell WC, Le Vine ES. Sexuality and pregnancy. A prospective study. J Reprod Med. 1982;27:321-7.

29. Basson R. Women's sexual dysfunction: Revised and expanded definitions. Canadian Med Assoc J. 2005;172(10):1327-33.

30. Tosun GF, Gördeles BN. Evaluation of sexual functions of the pregnant women. J Sex Med. 2014;11(1):146-53.

31. Cayan S, Akbay E, Boslu M, Canpolat B, Acar B. The prevalence of sexual dysfunction and potential risk factors that may impair sexual function in Turkish women. Urol Int. 2004;72:52-7.

32. Hanafy S, Srour NE, Mostafa T. Female sexual dysfunction across the three pregnancy trimesters: an Egyptian study. Sex Health. 2014;11(3):240-3.

33. Abouzari-Gazafroodi K, Najafi F, Kazemnejad E, Montazeri A. Demographic and obstetric factors affecting women's sexual functioning during pregnancy. Reproductive Health. 2015;12:2-5.

34. Ahmed MR, Madny EH, Sayed Ahmed WA. Prevalence of female sexual dysfunction during pregnancy among Egyptian women. J Obstet Gynaecol Res. 2014;40(4):1023-9.

Cite this article as: Anzaku SA, Ogbe EA, Ogbu GI, Edem BE, Ngwan SD. Evaluation of changes in sexual response and factors influencing sexuality during pregnancy among Nigerian women in Jos, Nigeria. Int J Reprod Contracept Obstet Gynecol 2016;5:3576-82 